\title{
Dust evolution of comet C/1995 01 (Hale-Bopp) by imaging polarimetric observations
}

\author{
E. Hadamcik ${ }^{1}$ and A. C. Levasseur-Regourd ${ }^{1,2}$ \\ 1 Service d'Aéronomie/CNRS, BP 3, 91371 Verrières le Buisson, France \\ 2 Université Paris VI, T15-15, Boîte 102, 4 place Jussieu, 75252 Paris Cedex 05, France
}

Received 28 August 2001 / Accepted 21 November 2002

\begin{abstract}
Comet $\mathrm{C} / 1995 \mathrm{O} 1$ (Hale-Bopp) has been observed over a large range of phase angles $\left(7^{\circ}-47^{\circ}\right.$ ) by the imaging polarimetric method from June 1996 to April 1997. Polarization maps were obtained and long-term evolution (month) of the coma structures followed. Short-term evolution $(20 \mathrm{~min}$ ) during the April 1997 observations has allowed us to measure the period of rotation of the central arc on the polarization maps. The comparison between the polarization maps and the intensity structures confirms a greater polarization in the jets. Phase curves are obtained for different regions of the coma and compared to those obtained for the whole coma. For the region around the nucleus, the phase curve is always underneath the phase curve corresponding to the whole coma, and the polarization at phase angles smaller than $25^{\circ}$ is negative $\left(P_{\min }=-5 \%\right)$. In the jets, the phase curve is always above the whole coma phase curve, and the polarization is always positive. Whole coma phase curves are obtained with all the available data sets in different wavelengths. A comparison with other diagnostics and laboratory experiments leads us to suggest that small grains build up fluffy aggregates of high porosity; the light scattered by such aggregates is highly polarized, as observed in this active comet.
\end{abstract}

Key words. comets: individual: C/1995 01 (Hale-Bopp) - polarization - scattering - ISM: dust, extinction techniques: polarimetric

\section{Introduction}

The light scattered by dust particles in comet C/1995 O1 (HaleBopp) has been intensively studied by various techniques (see e.g. Hanner et al. 1997; Jockers et al. 1997; Jorda et al. 1997), allowing some physical properties to be tentatively deduced by comparison with models. Amongst these techniques, observations of linear polarization have been performed. At phase angles greater than about $30^{\circ}$, the scattered light is found to be highly polarized as compared to comet $1 \mathrm{P} /$ Halley and more generally to comets belonging to the so-called high $P_{\max }$ comets in the Levasseur-Regourd et al. (1996) classification (see for example Kiselev et al. 1997; Ganesh et al. 1998; Manset \& Bastien 2000). On polarization maps, highly polarized regions correspond to jet-like and arc features (Hadamcik et al. 1997; Jockers et al. 1997; Jones et al. 1997; Furusho et al. 1999), as previously observed for 1P/Halley (Eaton et al. 1988), C/1990 K1 Levy (Renard et al. 1992), C/1996 B2 Hyakutake (Tozzi et al. 1997).

In the first part, the variations of the bright structures in the coma, both in intensity and polarization, is studied as a function of the phase angle $\alpha$ (at about $7^{\circ}, 19^{\circ}, 30^{\circ}$ and $45^{\circ}$ ), corresponding respectively to four observing periods (June 1996 and September-October 1996, February 1997, April 1997).

Send offprint requests to: E. Hadamcik, e-mail: edith.hadamcik@aerov.jussieu.fr
The spin axis of the nucleus, as derived from the coma morphology (Licandro et al. 1997), was lying close to the line of sight direction in April 1997. It thus allowed us to determine the period of rotation and the expansion speed of the dust arcs from the evolution of the structures in the polarization maps, as detailed in the second part.

The whole coma polarimetric phase curve is then compared to phase curves obtained for different regions inside the bright structures (jets and arcs) and in the circumnucleus region. After these three curves - corresponding to data obtained with the same instruments - are studied, the whole set of data already published is used to derive more documented synthetic phase curves for each wavelength for this quickly-evolving comet.

A comparison between the polarimetric results and those obtained by other techniques (dynamic of the particles in the jets, infrared spectroscopy) and by laboratory measurements is finally done, to provide a better understanding of the particles' physical properties.

\section{Observations and data reduction}

Our observations have been performed with the CCD imaging polarimetric technique (Renard et al. 1992, 1996) with a Cassegrain configuration from two telescopes: the $2 \mathrm{~m}$ at Pic-du-Midi Observatory and the $0.8 \mathrm{~m}$ at Haute-Provence Observatory (France). We used a $1024 \times 1024$ pixel TEK CCD, 


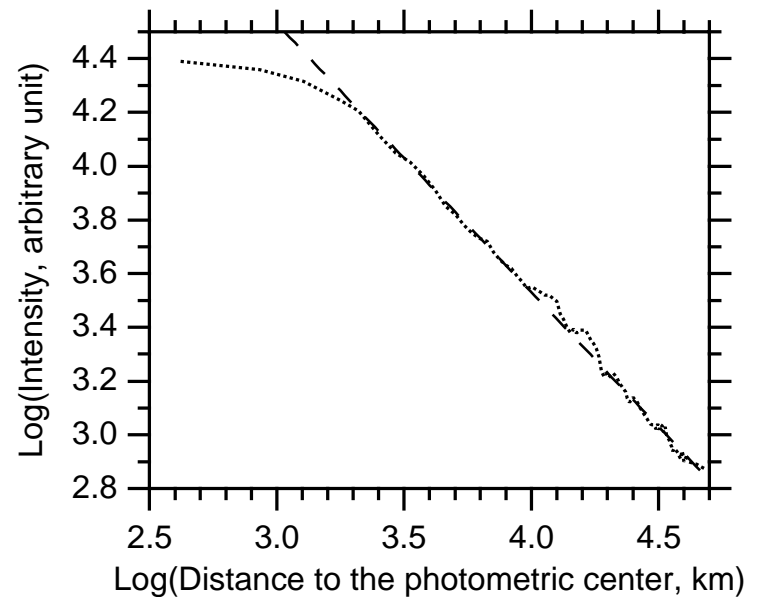

Fig. 1. Radial profile in intensity for April 9, 1997 observations. For distances larger than $2000 \mathrm{~km}$, slope $(-1.015 \pm 0.008)$. Some slight differences can be noticed near the arcs. Around the photometric center, the seeing flattens the curve.

with a resolution of 0.2 arcsec per pixel ( $2 \mathrm{~m}$ telescope), and a $512 \times 512$ pixel TEK CCD with a resolution of 0.42 arcsec per pixel ( $0.8 \mathrm{~m}$ telescope). To avoid any contribution of the emission lines of the gaseous species, different filters were used: in the red domain where contamination from gaseous emissions is negligible, a broad-band filter at $(670 \pm$ $50 \mathrm{~nm}$ ) was used; on the other hand, in the green domain where the gaseous emissions could be critical, a narrow-band filter centered on a region where the emissions are negligible at $(523 \pm 4 \mathrm{~nm})$ was used.

Four Polaroid filters were mounted on a rotating wheel, with their fast axis oriented at $45^{\circ}$ from one another. For each orientation, a polarized intensity image was recorded (so-called $Z_{0}, Z_{45}, Z_{90}, Z_{135}$ ). Short exposure times (in a $2 \mathrm{~s}$ to $120 \mathrm{~s}$ range) were chosen to ensure the stability of the sky background between two consecutive images. This also allows the use of startracking mode. The polarization of standard stars was also measured during each observational period.

Each individual image was flat field corrected. The sky background was first estimated in the outer part of the image in the sunward direction and subtracted. Intensity images were obtained by adding two individual images: either $\left(Z_{0}+Z_{90}\right)$ or $\left(Z_{45}+Z_{135}\right)$.

The two resulting images, if taken at less than $10 \mathrm{~min}$ interval, have to be identical. The radial profile (Fig. 1), with a slope of -1 in Log-Log scale, is a good test to better adjust the sky background. In April 1997, the comet was low on the horizon and a correction had to be applied from one image to the other. For each filter a linear correction of the image gives acceptable results (standard deviation between the polarization maps lower than $0.1 \%$ ) for projected apertures on the sky of around $50000 \mathrm{~km}$. Some discrepancies are noted for projected apertures smaller than $4000 \mathrm{~km}$ (standard deviation of the order of $0.5 \%$ ).

Maps of polarization $(\mathrm{P})$ are obtained by the combination of four consecutive individual polarized images in a same set, with

$P=2 \frac{\sqrt{\left(Z_{0}-Z_{90}\right)^{2}+\left(Z_{45}-Z_{135}\right)^{2}}}{Z_{0}+Z_{90}+Z_{45}+Z_{135}}$.

The four images are centered with a precision of 0.1 pixel. To assess the reality of the structures that are pointed out (low and high polarization regions), the polarization is measured by two methods. First, the average polarization through 12 pixels apertures (more than twice the seeing) is directly measured on the maps in the structures. Secondly, the four fluxes in each polarized image are measured through the same 12 pixel apertures and the polarization value is computed. A difference in the two polarization values would point out a lack of accuracy in the determination of the center of the images, meaning that the images have to be centered again.

With the $0.8 \mathrm{~m}$ telescope at Haute-Provence, the four polarized intensities are needed to retrieve the polarization. On the other hand, with the $2 \mathrm{~m}$ telescope at Pic-du-Midi, the polarized filters can be oriented with respect to the solar position angle with a precision of $1^{\circ}$. Then, $\left(Z_{45}=Z_{135}\right)$ and only two orientations of the filters are needed to obtain the polarization maps. Nevertheless, with the two telescopes, the images are recorded through the four filters. The comparison between $Z_{45}$ and $Z_{135}$ allows us to make a verification of the correct orientation of the filters; if $Z_{45}$ is different from $Z_{135}$, the four images are used to retrieve the polarization.

The Log of the observations is given in Table 1. For June 1996, September-October 1996 and April 1997 observations, maps of polarization are obtained. Whole coma polarization values, which correspond to a field which contain the major structures are retrieved for a series of polarized components from the integrated flux for all the nights, altogether with intensity images.

\section{Analysis of the results}

\subsection{Long-term evolution (June 1996 to April 1997)}

Polarization maps were retrieved on June 1996 near $\alpha=7^{\circ}$, on September 1996 near $\alpha=19^{\circ}$, and on April 1997 near $\alpha=45^{\circ}$. All the polarization maps and brightness images presented in Figs. 2, 3 and 4 were obtained through a red filter. Figure 2 shows the general evolution of the structures for the same projected distance of $150000 \mathrm{~km}$ on the Sun-comet line (this distance corresponding to a projected field that includes the main structures of the April observations). It allows a comparison for the same region of the coma, corresponding to different apertures for each observational period, depending on the distance to the Earth and on the phase angle. The bright structures (straight jets or/and arcs) are emphasized in the intensity images, once processed by the rotational gradient method (Larson $\&$ Sekanina 1984). The polarization values in the different regions are given in Tables 2 and 3. The results can be described as follows.

On June 12,1996 , at a phase angle $\alpha \cong 7^{\circ}$, (Fig. 2a) the whole coma polarization was equal to $(-0.4 \pm 0.4) \%$ in a circular field of $19600 \mathrm{~km}$. A lower polarization was measured around the photometric center, with an extrema of $(-5 \pm 1) \%$. 
Table 1. Conditions of observation of comet C/1995 01, $D=$ distance comet-Earth, $R=$ distance comet-Sun, Pos. $=$ Sun position angle, $m_{\mathrm{v}}=$ visual total magnitude, $h=$ elevation, seeing, exp. $t$. = exposure time for each individual image, $n=$ number of sets of individual images which allowed to establish the polarization ratios and maps ( 1 set $=2$ images at Pic du Midi and 4 images at OHP). Last column Red $=$ red filter and $\mathrm{G}=$ green filter.

\begin{tabular}{|c|c|c|c|c|c|c|c|c|c|c|}
\hline C/1995 O1 & $\begin{array}{c}\text { date } \\
\mathrm{h}: \min \mathrm{TU}\end{array}$ & $\begin{array}{c}\Delta \\
(\mathrm{AU})\end{array}$ & $\begin{array}{c}R \\
(A U)\end{array}$ & $\begin{array}{c}\alpha \\
\left({ }^{\circ}\right)\end{array}$ & $\mathrm{m}_{\mathrm{v}}$ & $\begin{array}{l}\text { Pos. } \\
\left({ }^{\circ}\right)\end{array}$ & $\begin{array}{l}\mathrm{h} \\
\left({ }^{\circ}\right)\end{array}$ & $\begin{array}{l}\text { seeing } \\
(\text { (') }\end{array}$ & $\begin{array}{l}\text { exp. t. } \\
\text { (s) }\end{array}$ & $n$ \\
\hline & $06 / 09$ & & & & & & & & & \\
\hline Pic du Midi & 01:05 & 3.3 & 4.2 & 7.5 & 7.2 & 110 & 32 & 1.0 & 120 & 4Green \\
\hline Observatory & to $01: 58$ & & & & & & & & & \\
\hline TBL (T2m) & $06 / 10$ & & & & & & & & & \\
\hline$F / D=25$ & $00: 59$ & 3.2 & 4.1 & 7.3 & 7.2 & 112 & 32 & 1.3 & 60 & 4Red \\
\hline Red filter: & to $02: 01$ & & & & & & & & & \\
\hline$(670 \pm 50) \mathrm{nm}$ & $06 / 12$ & & & & & & & & & \\
\hline \multirow{13}{*}{$\begin{array}{l}\text { Green filter: } \\
(523 \pm 4) \mathrm{nm}\end{array}$} & $00: 58$ & 3.2 & 4.1 & 6.9 & 7.2 & 113 & 31 & 1.6 & 60 & 4Green \\
\hline & to $01: 45$ & & & & & & & & & \\
\hline & $01: 49$ & 3.2 & 4.1 & 6.9 & 7.2 & 113 & 32 & & & \\
\hline & to $02: 48$ & & & & & & & 1.4 & 90 & 6Red \\
\hline & $09 / 30$ & & & & & & 24 & & & \\
\hline & $20: 08$ & 3.0 & 2.9 & 19.6 & 5.6 & 92 & $\downarrow$ & 0.8 & 60 & 3Red \\
\hline & to $21: 07$ & & & & & & 19 & & & \\
\hline & $10 / 03$ & & & & & & 27 & & & \\
\hline & $19: 38$ & 3.0 & 2.8 & 19.5 & 5.7 & 93 & $\downarrow$ & 2 & 60 & 4Red \\
\hline & to $20: 46$ & & & & & & 17 & & & \\
\hline & $02 / 06$ & & & & & & & & & \\
\hline & $06: 11$ & 1.9 & 1.3 & 29.3 & 2.4 & 146 & 28 & 2.0 & 10 & 2Red \\
\hline & to $06: 18$ & & & & & & & & & \\
\hline \multirow{24}{*}{$\begin{array}{c}\text { Haute } \\
\text { Provence } \\
\text { Observatory } \\
(\mathrm{OHP}) \\
\mathrm{T} 0.80 \mathrm{~m} \\
\mathrm{~F} / \mathrm{D}=15 \\
\text { Red filter: } \\
(650 \pm 70) \mathrm{nm} \\
\text { Green filter: } \\
(523 \pm 4) \mathrm{nm}\end{array}$} & $04 / 01$ & & & & & & 21 & & & \\
\hline & $19: 45$ & 1.35 & 0.9 & 47.4 & 0.0 & 153 & $\downarrow$ & 2.5 & 4 & 6 Red \\
\hline & to $20: 50$ & & & & & & 17 & & & \\
\hline & $04 / 02$ & & & & & & 23 & & & \\
\hline & $19: 30$ & 1.36 & 0.9 & 47.0 & 0.0 & 151 & $\downarrow$ & 2.5 & 2 & 6Red \\
\hline & to $20: 35$ & & & & & & 15 & & & \\
\hline & $04 / 03$ & & & & & & 22 & & & \\
\hline & $19: 51$ & 1.37 & 0.9 & 46.2 & 0.0 & 149 & $\downarrow$ & 4 & 60 & 5Green \\
\hline & to $21: 12$ & & & & & & 15 & & & \\
\hline & $04 / 05$ & & & & & & 22 & & & \\
\hline & $18: 55$ & 1.39 & 0.9 & 45.7 & 0.0 & 144 & $\downarrow$ & 6.5 & 4 & 4Red \\
\hline & to $20: 40$ & & & & & & 18 & & & \\
\hline & $04 / 06$ & & & & & & 30 & & & \\
\hline & $19: 25$ & 1.40 & 0.9 & 45.2 & 0.0 & 142 & $\downarrow$ & 3.5 & 2 & 7Red \\
\hline & to $20: 39$ & & & & & & 16 & & & \\
\hline & $04 / 07$ & & & & & & 26 & & & \\
\hline & $19: 30$ & 1.41 & 0.9 & 44.7 & 0.1 & 140 & $\downarrow$ & 5 & 4 & 4Red \\
\hline & to $21: 02$ & & & & & & 16 & & & \\
\hline & $04 / 09$ & & & & & & 26 & & & \\
\hline & 19:08 & 1.43 & 0.9 & 43.6 & 0.2 & 137 & $\downarrow$ & 4 & 2 & 9Red \\
\hline & to $20: 50$ & & & & & & 14 & & & \\
\hline & $04 / 10$ & & & & & & 29 & 3 & & \\
\hline & $19: 10$ & 1.44 & 0.9 & 43.2 & 0.2 & 135 & $\downarrow$ & $\downarrow$ & 60 & 4Green \\
\hline & to $20: 30$ & & & & & & 17 & 5 & & \\
\hline
\end{tabular}

This region near the nucleus has an elongated shape due to two higher polarization regions of $(2 \pm 1) \%$ in the solar and antisolar directions on June 9 and June 12. The maps retrieved through the green narrow-band filter (about one hour later) are similar. On the intensity images (Fig. 2b), the brightest region is perpendicular in projection to the Sun direction and five faint radial jets were observed. The brightest region does not appear on the polarization map. This seems to indicate a different population of dust in this region than in the other bright structures.

On September 30,1996 , at $\alpha \cong 19.6^{\circ}$, the whole coma polarization was equal to $(1.5 \pm 0.6) \%$ in a circular field of view of $50300 \mathrm{~km}$. On the polarization maps (Fig. 2c) a large fan with a higher polarization of $(4 \pm 1) \%$ corresponds to the straight radial bright jets on the intensity image. Seven smaller jets are observed (Fig. 2d). A tiny lower polarization region of $(-2 \pm 1) \%$ is observable in the direction opposite to the fan. The polarization decreases toward the Sun, while the jets are observed in the antisunward direction where the polarization is relatively high. On October 3, 1996, the whole coma polarization was of $(2.1 \pm 0.9) \%$, with a lower polarization region extending in the sunward direction and a higher polarization in the antisunward direction. The higher and lower values are the same as on September 30.

In April $1997\left(\alpha \cong 45^{\circ}\right)$, the whole coma polarization was in the interval $[15.2 \%, 13.4 \%]$, decreasing with the phase angle for a field of view in the 110000 to $103000 \mathrm{~km}$ range. The high polarization arcs on the maps have values of $(17 \pm 1) \%$ near the photometric center (Figs. 2e and 3a,c,e). Circumnucleus regions, on which the first arcs are superimposed, present a lower polarization with an average value of $(10 \pm 1) \%$. The bright 


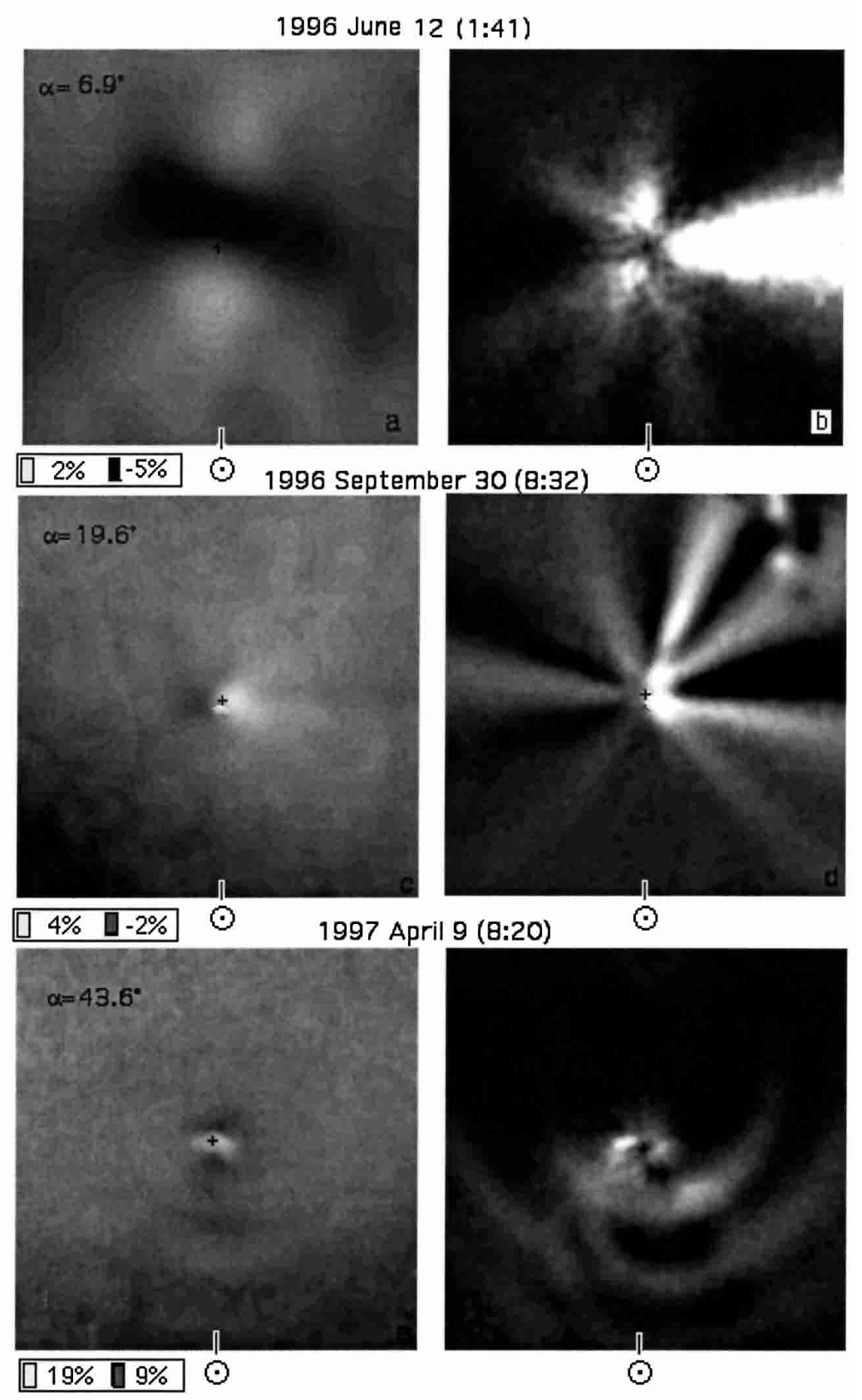

Fig. 2. Long-term evolution of the bright structures in polarization (left column) and in intensity (right column) from June 1996 to September 1996, and April 1997. The intensity images are treated by the rotational gradient method. The higher polarization is correlated to the bright structures except for the large June structure, which seems perpendicular to the Sun direction. Projected distances on the Sun-comet line of $150000 \mathrm{~km}$. arcs on the intensity images (Figs. $2 \mathrm{f}$ and $3 \mathrm{~b}, \mathrm{~d}, \mathrm{f}$ ) correspond to the high polarization arcs. A temporal evolution can be noticed within short intervals of time, for example from a comparison between Figs. 2e,f and 3e,f: the central arc departure was at about $60^{\circ}$ from the solar direction (Figs. 3e,f) and was at $90^{\circ}$ one hour later (Figs. 2e,f). This rotation of the central arc, near the nucleus, is pointed out both on the polarization maps and on the intensity images.

These observations confirm with a greater accuracy the correlation already noticed between the jets and arcs and the high polarization regions (Hadamcik et al. 1997). Nevertheless, some bright structures are found to result from a geometric effect instead of coming from the ejection or the confinement of particles. The existence of a low polarization circumnucleus region is confirmed at each phase angle, even if it is partially hidden by the presence of jets. For the first time, a highly negative polarization $(-5 \%)$, compared to the whole coma polarization $\left(-0.5 \%\right.$ at $\left.\alpha=8^{\circ}\right)$, is observed in the circumnucleus region at small phase angles. In the jets, the polarization is always positive. Phase curves for the different regions are derived (see Sect. 3.3.2).

\subsection{Short-term evolution (April 1 to 10,1997 )}

From April 1 to 10, 1997, the comet was observable each night, for about $1.5 \mathrm{~h}$. On the polarization maps and intensity images, the rotation of the central arc can be measured easily from observations performed during one single night (one sequence of four polarized images takes only $10 \mathrm{~min}$ ). In Fig. 4, the maps of polarization on April 9, taken at one-hour intervals, are 


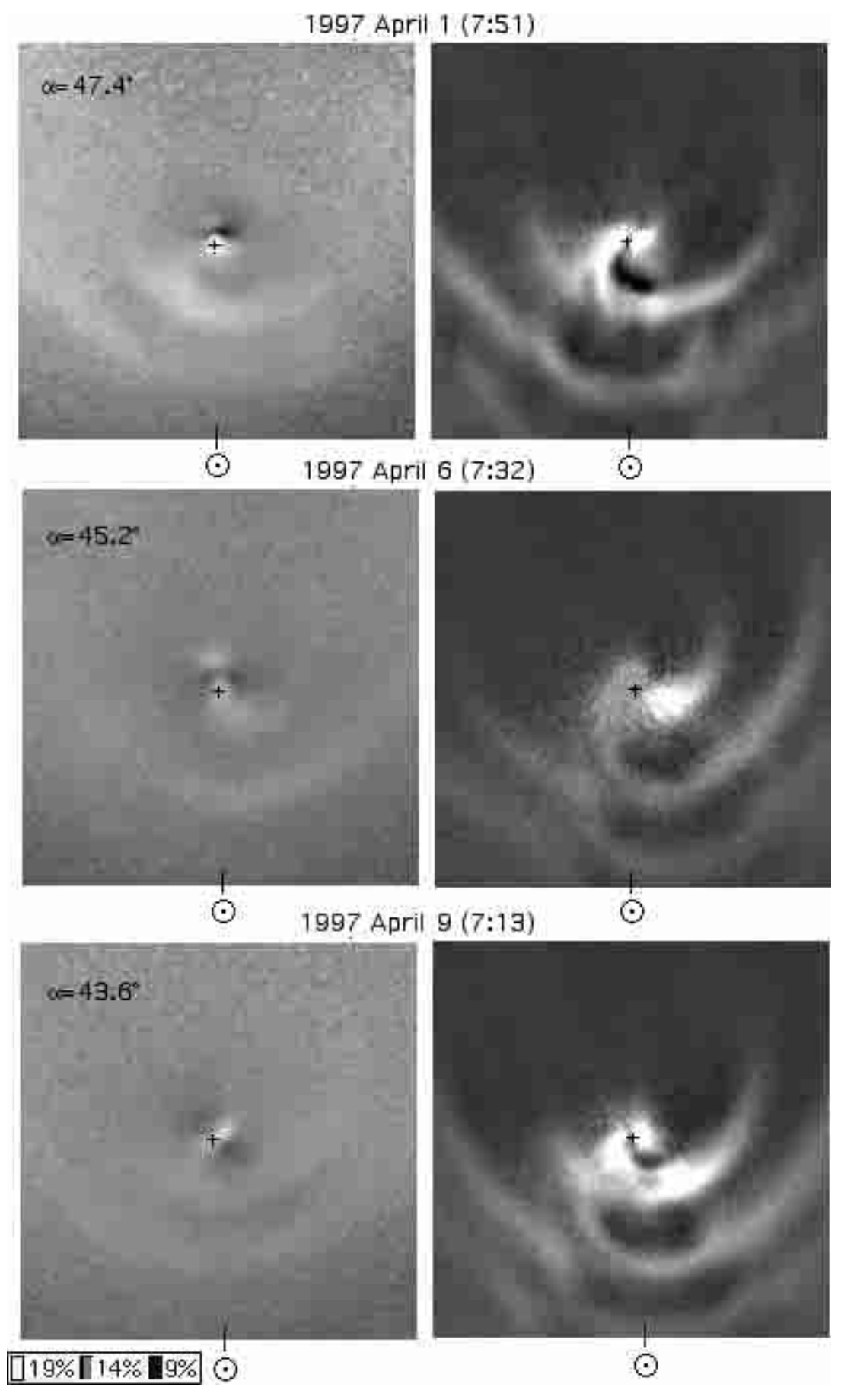

Fig. 3. Short-term evolution of the bright structures in polarization and intensity during the April 1997 observations. Note that the brightest regions on the arcs in the intensity images correspond to the superposition of two arcs sets. displayed in polar axes (horizontal axis for azimuth with the Sun direction at $+90^{\circ}$, and vertical axis for distances to the photometric center). The translation of the bright central arc is measured at $d=10$ pixels from the horizontal axis $(\cong 4000 \mathrm{~km})$. By using all the measurements, a rotation period of the central arc of $(11.2 \pm 0.5) \mathrm{h}$ is derived. This result compares well with Jorda et al. (1997), who found a period of rotation of $(11.34 \pm 0.03) \mathrm{h}$ from observations of the dust jets between February and March 1997.

The polarization value is stable inside the arcs while the intensity varies for all the April observations. Indeed, the brightest regions on the arcs, as seen on the intensity images, are the intersection of two series of bright arcs projected on the sky plane (see Fig. 3). The evolution in the arc position can be followed from one day to the other, both on the polarization maps and on the intensity images. The distance between the arcs remains constant. The average projected distance on the sky plane between two consecutive arcs is $(16500 \pm 500) \mathrm{km}$. The projected dust arc expansion velocity, of $(0.41 \pm 0.05) \mathrm{km} \mathrm{s}^{-1}$, is then derived from the value of the rotational period. This result is in good agreement with the values found by other observers using different techniques, i.e. $(0.41 \pm 0.02) \mathrm{km} \mathrm{s}^{-1}$ by Warell et al. (1997) in April 1997. 
Table 2. Polarization values in the different regions of the coma. The whole coma polarization is calculated for an aperture corresponding to a $150000 \mathrm{~km}$ projected distance on the comet-Sun direction. $P$ circ. lowest value of polarization in the circumnuclear region. $P$ "jet" higher value of polarization in the jets. Observation periods June 1996 and September 1997. The average error bar in the different structures is estimated to $1 \%$.

\begin{tabular}{ccccccc}
\hline$\alpha\left({ }^{\circ}\right)$ & 6.9 & 6.9 & 7.3 & 7.5 & 19.5 & 19.6 \\
filter & Red & Green & Red & Red & Red & Red \\
\hline field $\left(10^{3} \mathrm{~km}\right)$ & 18 & 18 & 19.1 & 19.5 & 50.1 & 50.3 \\
& & & & & & \\
P (\%) & & & & & & \\
whole coma & $-0.4 \pm 0.4$ & $-1.0 \pm 0.8$ & $-0.3 \pm 0.4$ & $-0.5 \pm 0.3$ & $2.1 \pm 0.9$ & $1.5 \pm 0.6$ \\
P "jet" & 2 & 1 & 2 & 1 & 4 & 4 \\
P circ. & -5 & -5 & -5 & -5 & -2 & -2 \\
\hline
\end{tabular}

Table 3. Same as Table 2 but for April 1997.

\begin{tabular}{|c|c|c|c|c|c|c|c|c|}
\hline $\begin{array}{l}\alpha\left({ }^{\circ}\right) \\
\text { filter }\end{array}$ & $\begin{array}{l}47.4 \\
\text { Red }\end{array}$ & $\begin{array}{l}47.0 \\
\text { Red }\end{array}$ & $\begin{array}{l}46.6 \\
\text { Green }\end{array}$ & $\begin{array}{l}45.7 \\
\text { Red }\end{array}$ & $\begin{array}{c}45.2 \\
\text { Red }\end{array}$ & $\begin{array}{l}44.7 \\
\text { Red }\end{array}$ & $\begin{array}{c}43.6 \\
\text { Red }\end{array}$ & $\begin{array}{l}43.2 \\
\text { Green }\end{array}$ \\
\hline field $\left(10^{3}\right) \mathrm{km}$ & 110 & 110 & 108 & 107 & 106 & 106 & 103 & 102 \\
\hline \multicolumn{9}{|l|}{$\mathrm{P}(\%)$} \\
\hline whole coma & $15.2 \pm 0.5$ & $15.8 \pm 0.9$ & $12.5 \pm 0.8$ & $14.4 \pm 0.5$ & $13.7 \pm 0.6$ & $13.2 \pm 0.9$ & $13.4 \pm 0.5$ & $10.9 \pm 0.9$ \\
\hline $\mathrm{P}$ central arc & 18 & 18 & 16 & 17 & 18 & 17 & 19 & 17 \\
\hline $\mathrm{P}$ arc 1 & 17 & 18 & 13 & 16 & 15 & 16 & 15 & 14 \\
\hline $\mathrm{P}$ arc 2 & 15 & 16 & 12 & 15 & 14 & 15 & 14 & 12 \\
\hline $\mathrm{P}$ circ. & 9 & 9 & 7 & 11 & 9 & 9 & 9 & 7 \\
\hline $\begin{array}{l}\mathrm{P} \text { between } \\
\text { arcs } 1 \text { and } 2\end{array}$ & 14 & 15 & 11 & 14 & 12 & 13 & 13 & 10 \\
\hline
\end{tabular}

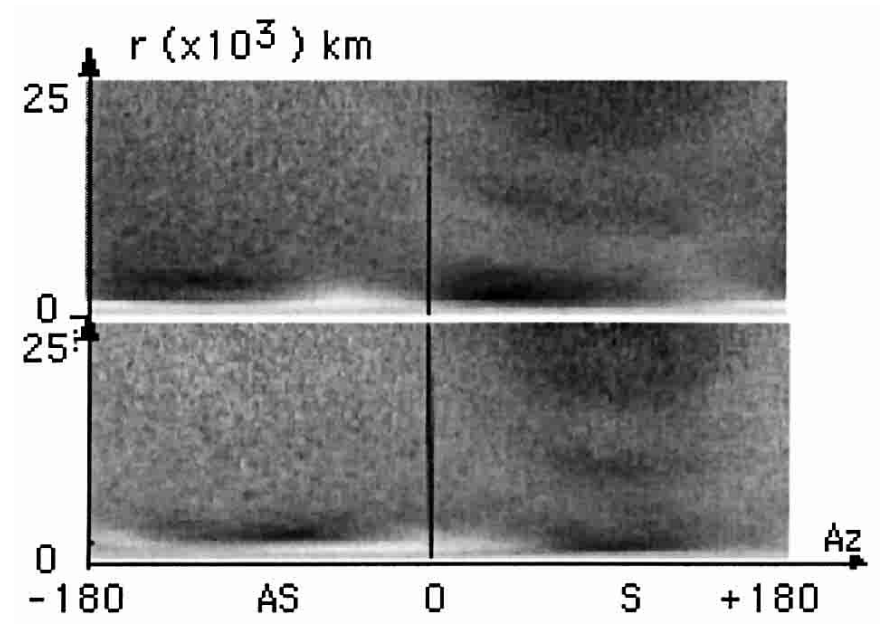

Fig. 4. Polarization maps in polar axes for April 9, 1997 (a. 19:20 UT, b. 20:20 UT): distances to the photometric center vs. azimuth. The origin for azimuths corresponds to the sunward direction on the right of the figure. The position of the central arc is measured at 10 pixels from the horizontal axis (about $4000 \mathrm{~km}$ ).

The evolution of the structures on the polarization maps has given some complementary measurements to deduce dynamical parameters of the dust. It also allows an accurate study of the evolution of the dust particles physical properties by the variation of the polarization inside the coma.

\subsection{Polarization variations in the coma}

\subsubsection{Profiles through the maps in the solar direction}

Cuts through the polarization maps and intensity images on the sunward direction emphasize the evolution of the dust properties inside the coma. In Fig. 5, all the profiles are presented with the same projected field within a diameter of $100000 \mathrm{~km}$; two vertical lines show the limit of the same projected coma region of $150000 \mathrm{~km}$ on the comet-Sun direction (which is the field on the Fig. 2 images). All the intensities are normalized at $5000 \mathrm{~km}$ in the Sun direction. Structures are compared from one day to the other, within the same observing period. On the polarization profiles, important variations can be noticed (up to $8 \%$ ), mainly in the circumnucleus region.

In Fig. 5a (June 1996), the polarization is highly negative in the central region (3000-4000 km), mainly in the antisunward direction. It increases up to 1 or $2 \%$ in the bright structures and is close to zero outside of them. In Fig. $5 b$, the intensity profiles are similar for the three nights, the small discrepancies resulting from the contribution of the faint jets in the sunward direction on June 9 and June 12. In Fig. 5c (September 1996), the higher polarization corresponding to the jets is superimposed on a lower polarization region. The general trend out of the central region (including the circumnucleus region and the central jet) is a slight increase in polarization from the sunward direction to the antisunward direction. The intensity profiles (Fig. 5d) are similar for the two nights, with a better seeing on September 30 than on October 3.

Only three profiles are presented, to clarify Figs. 5e and 5f for the April 1997 observations. In the circumnucleus region, the variation of polarization is important (up to 8\%). The general trend corresponds to an increase from the sunward direction to the antisunward direction (about $10 \%$ at $-50000 \mathrm{~km}$ to about $16 \%$ at $+50000 \mathrm{~km}$ ). Higher polarization values are generally observed on the sunward edge of the arcs (Fig. 6), with a decrease in polarization when the distance to the photometric center increases (a clue pointing to an evolution of the dust properties). In the antisunward direction, the particles pushed back from the arcs are likely to be progressively mixed with the particles directly ejected from the nucleus. 

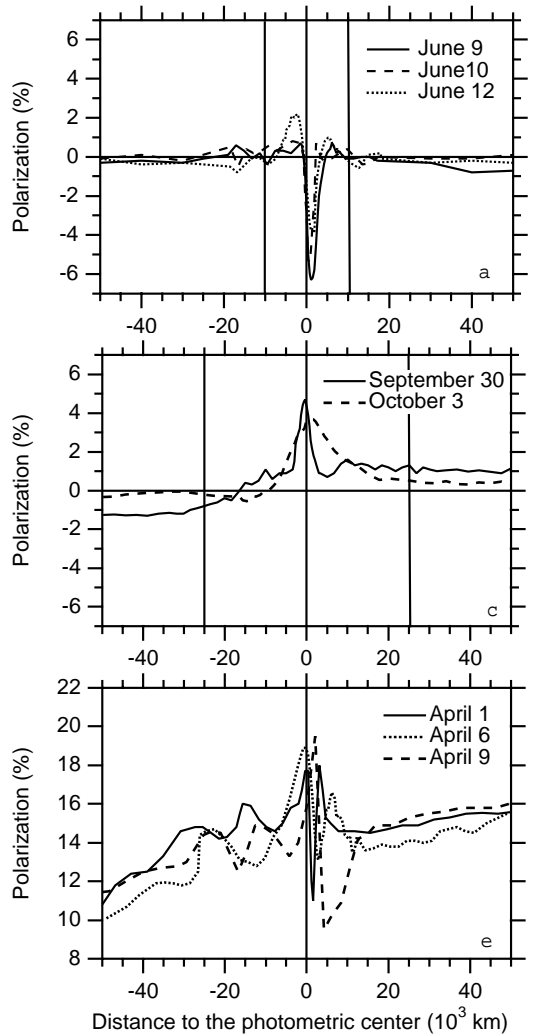
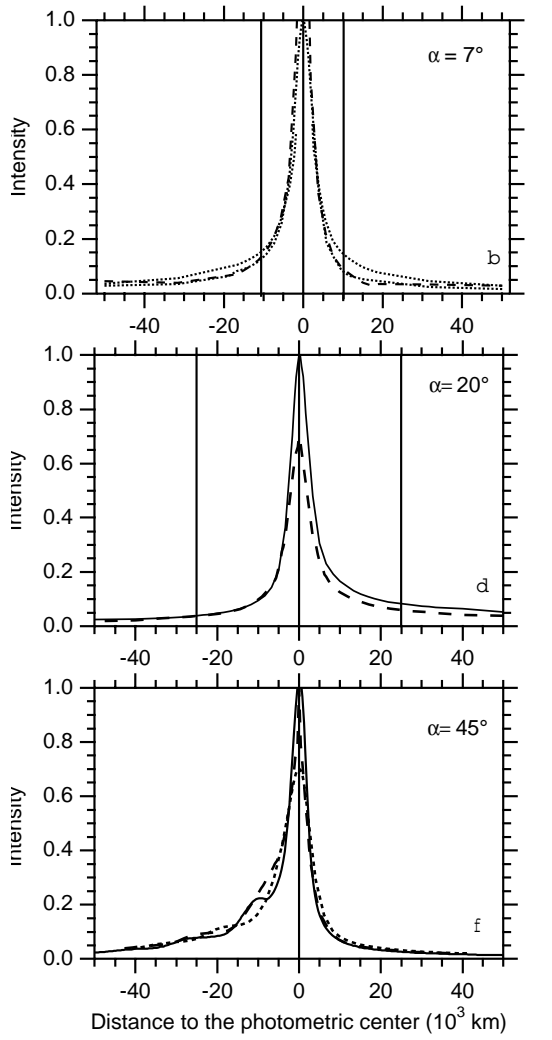

Fig. 5. Polarization and intensity profiles in sunward $(d<0)$ and antisunward $(d>0)$ directions, measured on the polarization maps and the intensity images. Averaged values on 6 pixel apertures. The field on projection had a diameter of $100000 \mathrm{~km}$. The two vertical lines are the limits of the same region of coma (projection on the Sun-comet line $150000 \mathrm{~km}$ as in Figs. 2 and 3). The structures are similar for all the observations at the same epoch. The intensities are normalized at $-5000 \mathrm{~km}$. The error bars are smaller than $0.5 \%$ in polarization and 0.02 (arbitrary unit) in intensity.
This process could increase the polarization. Another possibility could be the breaking of huge aggregates into smaller ones, which would also increase the polarization.

\subsubsection{Phase curves}

The whole coma polarization phase curve previously obtained for comet Hale-Bopp (Hadamcik et al. 1997) is used for comparison with the phase curves retrieved for the circumnucleus low polarization region and for the higher value region in jets and arcs (Fig. 7). The polarization value decreases when the dust in the arcs moves away from the nucleus. Between the two main arcs (1 and 2, which are further from the nucleus than the central arc), the polarization is almost identical to the integrated whole coma polarization. As expected at small phase angles (Levasseur-Regourd et al. 1996), the values corresponding to the green filter are close to those obtained with the red filter. Also, just as expected for $\alpha$ in the $10^{\circ}-47^{\circ}$ range, the polarization increases with the phase angle in all the regions. For phase angles near $45^{\circ}$, through the green filter, the polarization in all the regions (whole coma, jets, arcs and circumnucleus region) is lower than through the red filter, as already noticed for all comets. The parameters of the phase curves ( $\alpha_{\min }, P_{\min }, \alpha_{0}$ at inversion, $P_{45}$ at $\alpha=45^{\circ}$ ), deduced from trigonometric fits, are given for comparison between the different regions (Table 4$)$. The inversion angle for the whole coma curve is $\alpha_{0}=[12-0.5 /+6]^{\circ}$ and the minimum value of the polarization is $P_{\min }=(-0.4 \pm 0.2) \%$ at $\alpha_{\min }=(6 \pm 2)^{\circ}$. The inversion angle for the circumnucleus halo is $\alpha_{0}=(25 \pm 5)^{\circ}$ and the minimum value is $P_{\min }=(-5 \pm 2) \%$ at $\alpha_{\text {min }}=(8 \pm 2)^{\circ}, P$ being always lower than in the other regions. In the jets and arcs,
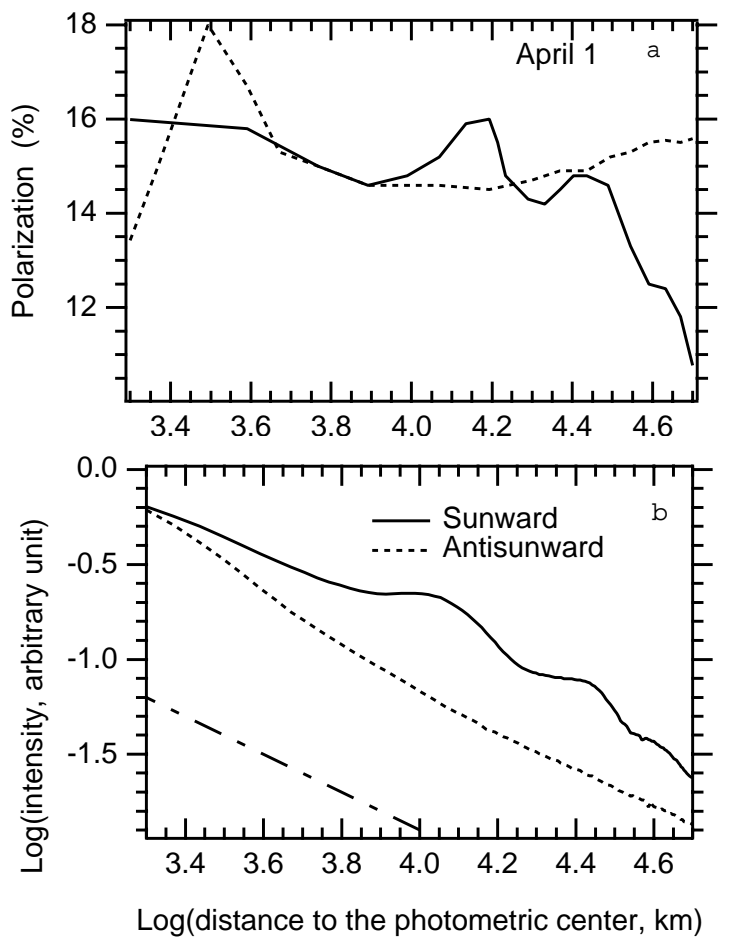

Fig. 6. Sunward and antisunward profiles vs. distance to the photometric center (in Log scale) for April 1, 1997. Average values on 6 pixel aperture. a) Polarization. The polarization decreases in the sunward direction at distances of about $40000 \mathrm{~km}$, and increases slowly in the antisunward direction. (error bars are smaller than $0.3 \%$ ). b) Intensity in Log-Log scale (error bars are about 0.05 at $10000 \mathrm{~km}$ and 0.1 at $40000 \mathrm{~km}$ ). 


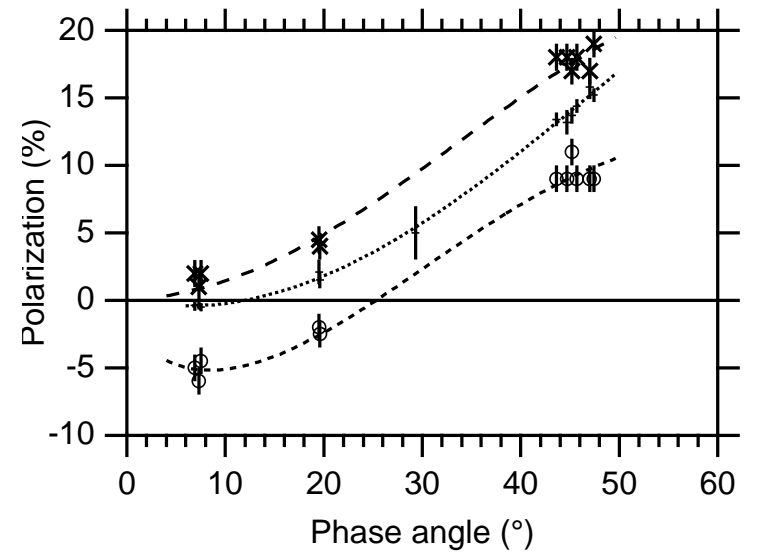

Fig. 7. Phase curves for an average wavelength of $(670 \pm 50) \mathrm{nm}$ : whole coma (+) fitted by a dotted line, circumnucleus region (o) fitted by a close dashed line and central arc $(\times)$ fitted by a large dashed line. Trigonometric fits.

the polarization is always positive (seeming to decrease progressively to 0 at $0^{\circ}$ ) and is higher than in the other regions. At $\alpha=45^{\circ}$, the maximum value is $P_{45}=(17.5 \pm 1.0) \%$ in the arcs and $P_{45}=(14.0 \pm 0.5) \%$ for the whole coma.

The spatial resolution of the polarization maps has allowed measurement of local values in particular regions where the dust properties are likely to be different. The difference noticed in the phase curves of these regions is indeed a clue to different dust properties. A comparison of our results to other observations and to laboratory measurements is now necessary.

\section{Discussion}

The whole coma phase curves obtained with all the published data set are first compared to whole coma polarimetric phase curves for other comets (Sect. 4.1). The wavelength dependence (Sect. 4.2) of the polarization leads to average physical properties of the dust particles. The different coma regions are then considered and the images and maps obtained in different wavelengths are discussed (Sect. 4.3). Other diagnostics such as brightness over a large range of wavelengths (from visible to submillimetric) or dynamics of the dust particles or infrared emission features are used to disentangle the physical properties considered to interpret the different polarization values (Sect. 4.4). Finally, laboratory measurements on light scattering by dust particles are used to define some physical properties of the particles.

\subsection{Phase curves}

To have an exhaustive phase curve, all the published data are used in Fig. 8 (data are from: Hadamcik et al. 1997; Jockers et al. 1997; Kiselev et al. 1997; Ganesh et al. 1998; Furusho et al. 1999; Manset \& Bastien 2000). The data may be compared to those obtained for 1P/Halley (Fig. 8a), which is a reference for cometary studies, as well as for the high $P_{\max }$ comets of the cometary polarimetric classification of Levasseur-Regourd et al. (1996, Fig. 8b). The phase curves
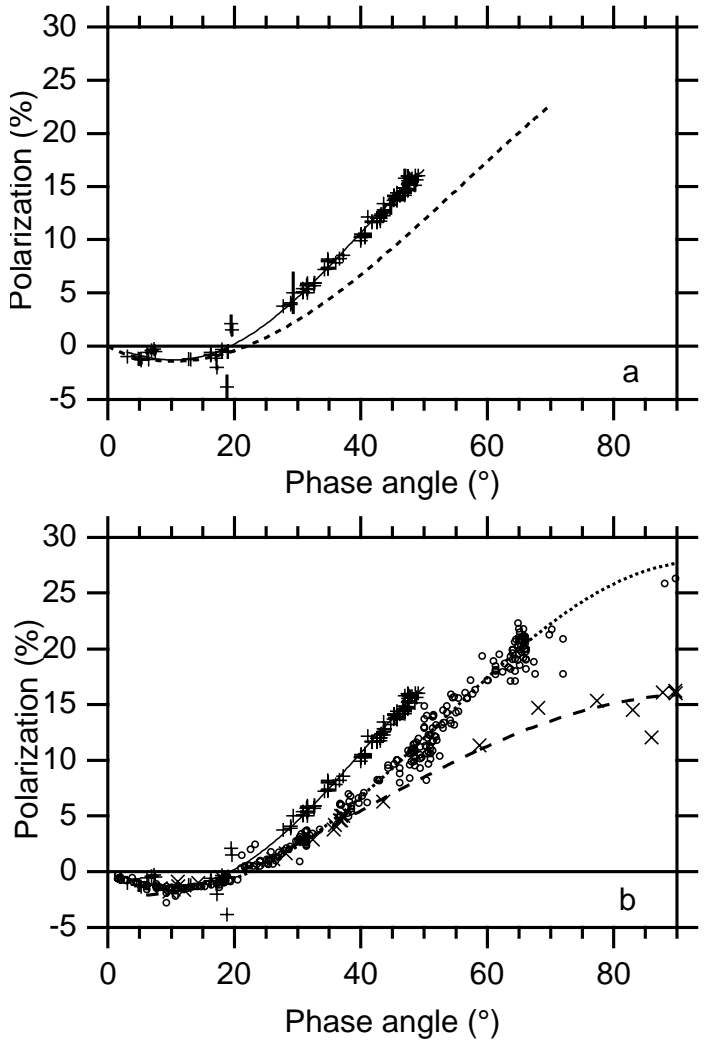

Fig. 8. a) Synthetic polarimetric phase curve (red domain) with all the published data. Comet Halley phase curve (dotted line) is given for comparison. b) Comparison between Hale-Bopp (+) and the other comets of the polarimetric classification: comets with high $P_{\max }$ (o), comets with low $P_{\max }(\times)$.

parameters for comets Hale-Bopp and Halley are compared in Table 5.

For phase angles smaller than $15^{\circ}$, the difference is small between all the abovementioned comets. The slope at inversion for comet Hale-Bopp $(0.28 \pm 0.02) \%$ is higher than for comet Halley $(0.24 \pm 0.02) \%$ in the red wavelength domain. It is of interest to notice that, although a higher slope is usually correlated to a smaller albedo (Umov 1905), the albedo retrieved from comparison between infrared thermal emission and visible scattered light (Williams et al. 1997) seems to be higher than for other comets. For phase angles between $25^{\circ}$ and $50^{\circ}$ a good agreement is found between the different observations. The whole coma polarization values for comet Hale-Bopp are higher than for other comets.

Near the inversion angle $\left(\alpha \cong 19^{\circ}\right)$, an important discrepancy is noticed between the observations, which take place over a significant time interval (about from September 4 to October 17), allowing important changes in the comet with jet activity. Our measurements on September 30 with $P=$ $(+1.5 \pm 0.6) \%$ and October 3 with $P=(+2.1 \pm 0.9) \%$ are significantly higher than the measurements by Manset \& Bastien (2000) with $P \cong(0.54 \pm 0.06) \%$ on September 4 and $(0.46 \pm$ $0.04)$ on October 15 and by Ganesh et al. (1998) with $P=$ $(-3.85 \pm 1.16) \%$ on October 17. This difference, which cannot be completely interpreted by apertures variations, could be the 
Table 4. Comparison of the whole coma phase curve parameters with those of the circumnucleus halo and of the jets and arcs. The data points are measured from our observations. The jet and arc polarization is always positive and decreases to 0 at $0^{\circ}$ phase angle.

\begin{tabular}{lllll}
\hline & $\alpha_{\min }\left({ }^{\circ}\right)$ & $\mathrm{P}_{\min }(\%)$ & $\alpha_{0}\left({ }^{\circ}\right)$ & $\mathrm{P}_{45}(\%)$ \\
\hline whole coma & $6 \pm 2$ & $-0.4 \pm 0.2$ & $12[-0.5,+6]$ & $14.0 \pm 0.5$ \\
circumnucleus halo & $8 \pm 2$ & $-5 \pm 2$ & $25 \pm 5$ & $9.0 \pm 1.0$ \\
jets and arcs & $(0)$ & $(0)$ & $(0)$ & $17.5 \pm 1.0$ \\
\hline
\end{tabular}

Table 5. Comparison of the whole coma phase curves parameters of comet Hale-Bopp and comet Halley in the red wavelength domain.

\begin{tabular}{llllll}
\hline & $\alpha_{0}\left({ }^{\circ}\right)$ & $\mathrm{h}\left(\% /{ }^{\circ}\right)$ & $\mathrm{P}_{\min }$ & $\alpha_{\min }\left({ }^{\circ}\right)$ & $\mathrm{P}_{50}(\%)$ \\
\hline Hale-Bopp & $19.2 \pm 0.5$ & $0.28 \pm 0.02$ & $-1.3 \pm 0.5$ & $10 \pm 2$ & $16.6 \pm 0.5$ \\
Halley & $22.0 \pm 0.5$ & $0.24 \pm 0.02$ & $-1.4 \pm 0.2$ & $10 \pm 1$ & $11.9 \pm 0.5$ \\
\hline
\end{tabular}

result of the high activity of the comet. Between September 25 and 31, Tozzi et al. (1997) have indeed observed an important outburst, which could correspond to the emission of jets leading to a higher polarization. Similarly, during the outburst of comet Halley on October 1985, the polarization had increased of about $3 \%$; it was interpreted by smaller sizes for the freshly emitted dust (Dollfus et al. 1988). The higher albedo and higher polarization could then be a clue to the presence of smaller grains.

\subsection{Wavelength dependence}

From all the observations presented here, the whole coma polarization, at phase angles higher than $30^{\circ}$, increases with the wavelength, as already noticed by various authors (i.e. Jockers et al. 1997; Kiselev \& Velishko 1997; Levasseur-Regourd 1999). All the phase curves have a similar behavior. Figure 9 presents the available data set (data from Hasegawa 1997, and Jones et al. 2000 for the infrared domain). The Jones et al. (2000) values at $\alpha \cong 40^{\circ}$, in the near infrared domain $(2000 \mathrm{~nm})$, are just below the fit obtained for the red domain and 5\% smaller than the values obtained by Hasegawa et al. (1997). This discrepancy could be due to the fact that they used different apertures (respectively 18.4 arcmin and 20 arcsec), and that the arcs region was thus not completely included in the Jones et al. observations.

Figure 10 presents the polarization as a function of the wavelength for two phase angles $\left(40^{\circ}\right.$ and $\left.50^{\circ}\right)$. Comet Halley measurements are also given for comparison. In the visible domain, the polarization value increases with the wavelength. It is more pronounced than for comet Halley. A maximum seems to appear at $(1600 \pm 200) \mathrm{nm}$. This could be interpreted as a higher limit for the size of the particles of the order of $0.5 \mu \mathrm{m}$ (Jones et al. 2000). Nevertheless, for wavelengths higher than $1600 \mathrm{~nm}$, a possible contamination by the cometary thermal emission could also occur, leading to a decrease in polarization. The distribution of the grains between the different coma regions also needs to be addressed.

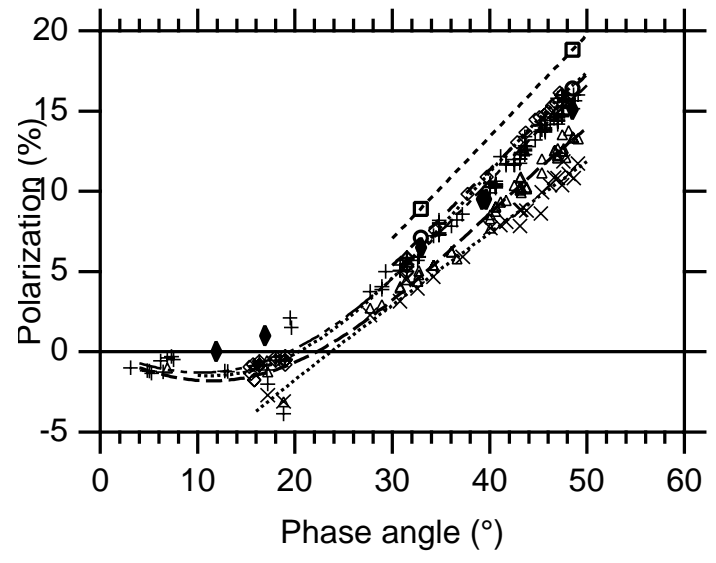

Fig. 9. Polarimetric color effect for the whole coma. Wavelength domains: $(\times) 365 \pm 4 \mathrm{~nm},(\triangle) 482 \pm 5 \mathrm{~nm},(+) 685 \pm 25 \mathrm{~nm},(\diamond) 740 \pm$ $5 \mathrm{~nm},($ o) $1240 \pm 85 \mathrm{~nm},(\square) 1650 \pm 150 \mathrm{~nm},(\diamond) 2200 \pm 75 \mathrm{~nm}$. The $2200 \mathrm{~nm}$ phase curve is not drawn to simplify the figure.

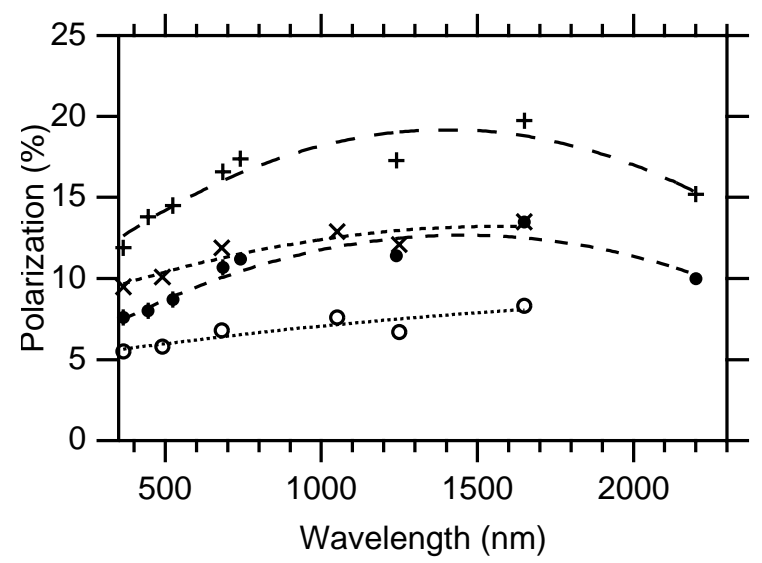

Fig. 10. Variation of polarization vs. wavelength at $\alpha=40^{\circ}$ (•) and $50^{\circ}(+)$. Same for comet Halley (o) and ( $\times$ ). Polynomial fits.

\subsection{Comparison with other polarization maps of comet Hale-Bopp}

The April 1997 polarization maps are in complete agreement with those of Jockers et al. (1997) and Furusho et al. (1999) in the visible domain. The polarization in the arcs decreases when the dust moves away from the nucleus. The Jones et al. (2000) maps of polarization, in the near infrared domain, present the 
same trends, with a polarization value higher in arc 1 and lower in arc 2, as compared to the central arc. The authors invoke some breaking of the particles with time between the central arc and arc 1. The different evolution for the two wavelength domains is not likely to be explained by this approach. A dilution of the particles in the background or a more important effect of the solar radiation pressure on the smallest particles could eventually explain the decrease of polarization in the arcs further away from the nucleus. Nevertheless, the higher value of polarization found on the leading edges of the two arcs (in Jones et al. and in our observations) suggests smaller grains in this region, expanding with the same velocity as all the dust inside the arcs. It seems to exclude the hypothesis of segregation by solar radiation pressure inside the arcs.

Jockers et al. (1997) and Furusho et al. (1999) have also studied the color effect on their intensity images. A color, bluer in the jets than in the other regions of the coma is noticed, confirming the presence of smaller grains in the arcs than in the surrounding coma. In summary, a size distribution with numerous Rayleigh grains could be a possible interpretation of the high polarization in the arcs.

As far as the circumnucleus region is concerned, lower polarization values are found on all our polarization maps with jets superimposed on it, as well as on the polarization maps of Jockers et al. (1997) or Jones et al. (2000). Jockers et al. obtained a smaller ratio between the polarization in the red domain and in the green domain (hereafter named bluer polarization color), in a region extending $10^{4} \mathrm{~km}$ (out of the superimposed central arc). This result agrees with the higher polarization in the blue-green domain observed in-situ in the near nucleus region $\left(10^{4} \mathrm{~km}\right.$ large $)$ of comet $1 \mathrm{P} /$ Halley by the OPE light-scattering experiment aboard the Giotto spacecraft (Hadamcik 1999). In this circumnucleus region an increase of the scattered intensity is also observed (Levasseur-Regourd et al. 1999). Eaton et al. (1988) also found a similar result $\left(P_{\text {blue }}>P_{\text {red }}\right)$ with remote observations of $1 \mathrm{P} /$ Halley, in a central aperture of $6300 \mathrm{~km}$, whereas the polarization through the red filter was also higher in the external region.

The size distribution was mainly invoked to interpret the different polarimetric observations, but the albedo and the porosity are also important parameters for polarization and its wavelength dependence. Numerous Rayleigh grains seem to be a possible interpretation. Nevertheless, it is necessary to compare with the results of other diagnostics to develop the interpretation of the observations.

\subsection{Comparison with other diagnostics}

To interpret the polarimetric observations in terms of physical properties of the particles, it is necessary to compare them to other diagnostics: albedo derived from the observations in near infrared (wavelength range $1 \mu \mathrm{m}-2.2 \mu \mathrm{m}$ ), temperature derived from the Planck function and superheat defined as the ratio of the color temperature and of the black body temperature in the thermal infrared $(\lambda>3 \mu \mathrm{m})$, silicate emission features strength near $10 \mu \mathrm{m}$, emissions in the submillimetric domain, dynamics of the particles by the spatial evolution of brightness. Associated with models, the physical properties involved are the size of the grains, the size and porosity of aggregates and the chemical composition (silicate and carbonaceous compounds).

As already mentioned in Sect. 4.1, Hale-Bopp seems to have a higher albedo than other comets. Williams et al. (1998) had interpreted this high albedo as indicating the presence of a large number of very small particles, and Hanner et al. (1998) as an increase of the ratio between silicates and carbonaceous compounds. The small grains are eventually included in fluffy aggregates of porosity higher than $95 \%$ as suggested by Greenberg \& Li (1999). These hypothesis have also been studied in laboratory measurements, and they are in agreement as can be seen below.

The grains of comet Hale-Bopp have higher color temperature than those of other comets at the same heliocentric distance (Mason et al. 2001). Thermal images in the $5 \mu \mathrm{m}-18 \mu \mathrm{m}$ wavelengths range (Hayward et al. 2000) show the same jets and arcs than in visible wavelengths. The important superheat and the high polarization could be due to particles of high porosity, rich in carbonaceous compounds and/or with a small mean grain size.

Well-structured silicate emission features at wavelengths near $10 \mu \mathrm{m}$ have been observed for Hale-Bopp (Crovisier et al. 1997; Hayward \& Hanner 1996; Hanner et al. 1997). They require grains size smaller than $1 \mu \mathrm{m}$; if these small grains are included in fluffy aggregates, the porosity has to be higher than 95\% (Hanner et al. 1987). Galdemard et al. (1997) have found a variation of the silicate features shape from one arc to another and have interpreted it as a fragmentation of particles, just like Jones et al. (1999) from their polarization maps. However, this interpretation is disputable since there is no increase in the polarization from one arc to the next in the visible domain. As seen before, the dust arcs have a constant expansion velocity value; this is also in opposition to a significant fragmentation.

To model the bright structures, a velocity higher for small particles and a higher effect of the radiation pressure are invoked. The expected outflow velocities of the dust seem to be confirmed by the hypothesis of the presence of a large number of submicron particles (Hayward et al. 2000). Nevertheless, as calculated by Nakamura et al. (1994), the gas drag forces on fractal aggregates of high porosity are roughly two orders of magnitude larger than on compact particles of the same mass. As a consequence, two kinds of particles could be confined in the jets and arcs: very small submicron particles or highly porous fluffy aggregates made of submicronic constituent grains. Fulle et al. (2000) have modeled the local properties of the light scattered by comet Halley dust particles during the Giotto encounter and the dust impact experiment aboard the same spacecraft; their best fit is for particles with densities of about $100 \mathrm{~kg} \mathrm{~m}^{-3}$ and thus very high porosity. The porosity of the particles could be even higher in Hale-Bopp to increase the polarization. The mapping of the color temperature has allowed Mason et al. (2001) to detect a segregation of particles through the coma (particles smaller than $0.1 \mu \mathrm{m}$ cannot be detected). In the sunward direction, for these authors, the dominating size seems to be smaller than at higher distances 
where more massive particles should dominate the distribution. In the antisunward side (tail side) smaller and less massive particles should dominate. The observed increase of polarization from the sunward direction to the antisunward direction is in accordance with this result.

Finally, Jewitt and Matthews (1999) have studied submillimeter continuum images. Their derived dust production rates seem to indicate that the dust emitted from the nucleus is dominated by large particles (millimeter sized). They are visually not taken into account, since the scattering cross-section (in the visible domain) of such large particles is very low. Nevertheless large porous fluffy aggregates could also induce high polarization values. All the above mentioned hypotheses may be assessed by laboratory measurements.

\subsection{Interpretation by light scattering laboratory measurements}

Two types of laboratory experiments deal with light scattering by aggregates. The microwave analog facility, at the University of Florida, studies single scattering by particles of various materials, shapes, porosities and sizes. The wavelengths (2.7 to $4 \mathrm{~mm}$ ) simulate the $430-660 \mathrm{~nm}$ visual domain, which includes the wavelengths used in cometary dust observations (Gustafson \& Kolokolova 2000). The PROGRA ${ }^{2}$ experiment (Worms et al. 1999) studies the red and/or green light scattered by levitating particles in microgravity conditions. It also operates under normal gravity with highly porous particles lifted by an air draught (Hadamcik 1999).

With the microwave analog experiment, light scattering observations have been correlated with some physical properties of the cometary dust. An increase of polarization (in the positive branch) together with a red polarization color (case of the whole coma) requires an increase in the imaginary part of the refractive index (more carbonaceous compounds), but leads to a decrease of the albedo. An increase of the polarization, together with a less red color and a redder polarization color (case of the arcs) could be due to a decrease in the size of the constituent grains or to a decrease of the compactness in the aggregates. The negative branch is more pronounced (case of the circumnucleus region) for more compact and absorbing particles.

With the PROGRA ${ }^{2}$ experiment, light scattering by mixtures of large fluffy aggregates of fractal type, with a porosity higher than $95 \%$ and constituent grains size of the order of $0.1 \mu \mathrm{m}$, have been found to reproduce the cometary whole coma phase curves (Hadamcik et al. 2001). As observed in the whole coma and in the arcs, the red polarization color increases if the grains are mixed with a small amount of carbon grains (1/1000 in mass), but disappears if the proportion of carbon is higher (1/100 in mass). The positive polarization observed in the case of jets and arcs could thus correspond to a high fluffiness of the particles acting as Rayleigh scatterers. The decrease of polarization in the circumnucleus region (at large phase angles) could be related to a lower porosity of the particles.

The fluffy aggregates studied with the PROGRA ${ }^{2}$ experiment are good candidates to simulate the high polarization and the polarimetric color in the jets and arcs. Their constituent grains, linked together in chains (overlapping) and creating small fluffy aggregates (like wool-waste) interact with light as larger grains. The high porosity corresponds to a high surface area with chains linked in flakes (like snow). Such particles are in the $1 \mu \mathrm{m}$ to $1 \mathrm{~mm}$ size range, with constituent grains in the $10 \mathrm{~nm}$ to $100 \mathrm{~nm}$ range. In the visible domain, the particles can be optically thin; in the infrared the small fluffy aggregates can play the role of constituent grains; in the submillimeter domain the whole aggregate is detected. The high polarization in the jets and arcs (without any negative branch) can be the result of an enrichment in submicronic grains (Rayleigh scatterers), included or not in highly fluffy aggregates. In the circumnucleus region, the particles could be more compact with an enriched composition of organic compounds, leading to a highly negative branch at small phase angles and a smaller polarization at large phase angles. The fluffy structures in the coma are the result of the evaporation of organic compounds.

\section{Conclusion}

The whole coma polarization of comet Hale-Bopp is higher than for other comets. The polarization color effect is important, with possibly a maximum polarization in the near infrared $(1600 \mathrm{~nm})$. A negative branch is noticed for phase angles below $19^{\circ}$. Three regions are systematically pointed out on the polarization maps: the jets and arcs, the circumnucleus region and the surrounding coma. The bright structures noticed at small phase angles in the intensity images have two different origins: the so-called jets (with a polarization higher than in the surrounding coma), and structures which could correspond to the projection of the elongated coma on the sky plane. The systematic correlation found between jets or arcs and high polarization regions allows us to determine the period of rotation of the central arc, $(11.2 \pm 0.5) \mathrm{h}$, and the velocity of the arcs, $(0.41 \pm$ $0.02) \mathrm{km} \mathrm{s}^{-1}$. In the jets the polarization is always positive in the visible wavelength domain. In the circumnucleus region, the minimum value of polarization can reach $-5 \%$, and is still $-2 \%$ at a $20^{\circ}$ phase angle.

The higher polarization of the dust coma of comet HaleBopp, as compared to that of other comets, suggests the existence of smaller grains included in highly porous large aggregates, and possibly an increase of the number of (high albedo) "white" grains in these aggregates. In the circumnucleus region, the particles are likely to be more compact. In the jets, the grains seem to be smaller than in the surrounding regions and/or the aggregates are fluffier with less carbonaceous compounds.

To have a more precise knowledge of the physical properties of the cometary dust particles, more polarimetric observations, with spatial resolution, are necessary over a large range of wavelengths (from the near ultraviolet to the near infrared). Studying the different regions with a spectropolarimeter would provide a more precise determination of the wavelength dependence of the polarization in the coma. As for laboratory measurements, the study of an extended number of levitating aggregates such as cometary analogues (silicates with different carbonaceous compounds) is essential. 
Acknowledgements. The authors thank all the teams at the Pic-du-Midi Observatory and at the Haute-Provence Observatory. They are grateful to J.B. Renard for his comments and to the participants of the "Workshop on polarization 2001 with emphasis on cometary observations", which took place at the Observatoire de Paris in April for fruitful discussions: P. Bastien, R. Furusho, B.A.S. Gustafson, M.S. Hanner, K. Jockers, T.J. Jones, S. Kikuchi, L. Kolokolova, K. Lumme, E.V. Petrova and G.P. Tozzi.

\section{References}

Crovisier, J., Leech, K., Bockelée-Morvan, D., et al. 1996, Science, 275, 1904

Dollfus, A., Bastien, P., Le Borgne, J. F., Levasseur-Regourd, A. C., \& Mukai, T. 1988, A\&A, 206, 348

Eaton, N., Scarrott, S. M., \& Warren-Smith, R. F. 1988, Icarus, 76, 270

Fulle, M., Levasseur-Regourd, A. C., McBride, M., \& Hadamcik, E. 2000, AJ, 119, 1968

Furusho, R., Suzuki, B., Yamamoto, N., et al. 1999, PASJ, 51, 367

Ganesh, S., Joshi, U. C., Baliyan, K. S., \& Deshpande, M. R. 1998, A\&A, 129, 489

Galdemard, P., Lagage, P. O., Dubreuil, D., et al. 1997 (published in 1999), Earth, Moon, Planets, 78, 271

Greenberg, J. M., \& Li, A. 1999, Space Sci. Rev., 90, 149

Hadamcik, E., Levasseur-Regourd, A. C., \& Renard, J. B. 1997 (published in 1999), Earth, Moon, Planets, 78, 365

Hadamcik, E. 1999, Ph.D. Thesis, Université Paris 6

Hadamcik, E., \& Levasseur-Regourd, A. C. 2000, C.R. Acad. Sci. Paris, 2B, C 2-316

Hadamcik, E., Renard, J. B., Worms, J. C., Levasseur-Regourd, A. C., \& Masson, M. 2001, Icarus, 155, 497

Hanner, M. S., Tokunaga, A. T., Golish, W. F., Griep, D. M., \& Kaminski, C. D. 1987, A\&A, 187, 653

Hanner, M. S., Gehrz, R. D., Harker, D. E., et al. 1997 (published in 1999), Earth, Moon, Planets, 79, 247

Hanner, M. S. 1999, Space Sci. Rev., 90, 99
Hasegawa, H., Ichikawa, T., Abe, S., et al. 1997 (published in 1999), Earth, Moon, Planets, 78, 353

Hayward, T. L., \& Hanner, M. S. 1996, Science, 275, 1907

Hayward, T. L., Hanner, M. S., \& Sekanina, Z. 2000, ApJ, 538, 428

Jewitt, D., \& Matthews, H. 1999, AJ, 117, 1056

Jockers, K., Rosenbush, V. K., Bonev, T., \& Credner, T. 1997 (published in 1999), Earth, Moon, Planets, 78, 373

Jones, T. J., \& Gehrz, R. D. 2000, Icarus, 143, 338

Jorda, L., Rembor, K., Lecacheux, J., et al. 1997 (published in 1999), Earth, Moon, Planets, 77, 167

Kiselev, N. N., \& Velichlo, F. P. 1997 (published in 1999), Earth, Moon, Planets, 78, 347

Larson, S., \& Sekanina, Z. 1984, AJ, 89, 571

Levasseur-Regourd, A. C., Hadamcik, E., \& Renard, J. B. 1996, A\&A, 313,327

Levasseur-Regourd, A. C., McBride, N., Hadamcik, E., \& Fulle, M. 1999, A\&A, 348, 636

Levasseur-Regourd, A. C. 1999, Space Sci. Rev., 90, 163

Licandro, J., Bellot Rubiot, L. R., Casas, R., et al. 1997 (published in 1999), Earth, Moon, Planets, 77, 199

Manset, N., \& Bastien, P. 2000, Icarus, 145, 203

Mason, C. G., Gehrz, R. D., Jones, T. J., et al. 2001, ApJ, 549, 646

Nakamura, R., Kitada, Y., \& Mukai, T. 1994, Planet. Space Sci., 42, 721

Renard, J. B., Levasseur-Regourd, A. C., \& Dollfus, A. 1992, Ann. Geophys., 10, 288

Renard, J. B., Hadamcik, E., \& Levasseur-Regourd, A. C. 1996, A\&A, 316, 263

Tozzi, G. P., Cimatti, A., di Serego Alighieri, S., \& Cellino, A. 1997 (published in 1999), Planet. Space Sci., 45, 535

Tozzi, G. P., Manucci, F., \& Stanga, R. 1997 (published in 1999), Earth, Moon, Planets, 78, 279

Umov 1905, Phys. Z., 6, 674

Warell, J., Lagerkvist, C. I., \& Lagerros, J. V. 1997 (published in 1999), Earth, Moon, Planets, 78, 197

Worms, J. C., Renard, J. B., Hadamcik, E., Levasseur-Regourd, A. C., \& Gayet, J. F. 1999, Icarus, 142, 281 